\title{
COMMUNICATION
}

\section{Snapshots of Modern Mathematics from Oberwolfach: Writing about Mathematics for a Wide Audience}

\section{Carla Cederbaum and Andrew Cooper}

Each week, renowned mathematicians meet at the Mathematisches Forschungsinstitut Oberwolfach (MFO) in Germany to discuss current research topics and develop new mathematical ideas. In 2013, the MFO, under its director Gerhard Huisken, started a project making the topics discussed at the MFO accessible to a wider audience, beyond the mathematical community. This project is called snapshots of modern mathematics from Oberwolfach.

\section{The Goals of the Snapshot Project}

The snapshot project has the goal of communicating modern mathematical research to what we call a wide audience-mathematics teachers, mathematically interested high school students, science journalists, and more generally those outside of the world of mathematical research who have an interest in understanding what is going on inside of it. We aim to demonstrate through many different examples that mathematics and mathematical research are fascinating, elegant, and diverse. The snapshots illustrate and explain the manifold applications of mathematics to other scholarly disciplines, and at the same time present and illuminate the character of the different areas of mathematics.

The diversity of authors and their perspectives on mathematics allow readers to gain an impression of

Carla Cederbaum is senior editor of the snapshots project. She is junior-professor in the mathematics department at the University of Tübingen in Germany. Her email address is cederbaum@ mfo.de.

Andrew Cooper is snapshots junior editor. He holds the post of teaching assistant professor in the department of mathematics at North Carolina State University. His email address is andrew. cooper@math.ncsu.edu.

For permission to reprint this article, please contact:

reprint-permission@ams.org.

DOI: http://dx.doi.org/10.1090/noti1407 what mathematicians strive for, and what motivates and inspires them. We want to show how mathematicians think and which ideas they find especially fascinating. Most of all, though, we want to make the readers curious about modern mathematics and mathematical research and maybe even encourage some students to study mathematics.

We also have the goal of supporting the mathematical community in communicating suitable research results and methods to a wide audience. As one snapshot author described his experiences participating in the project: "When writing a snapshot, one is forced to leave the mathematical 'autism' and to look at the content from a totally new perspective."

\section{The Snapshots}

Selected volunteers from each of the weekly scientific programs at the MFO write short snapshots of 8-12 A5 pages explaining suitable topics from their respective research areas as accessibly and understandably as possible without assuming much previous knowledge. The snapshots are then edited by a team of junior editors. These editors are students and researchers in mathematics. They are especially experienced in simplifying complex issues and bridging the gaps of knowledge to reach mathematics teachers and high school students.

After scientific refereeing by the organizers of the respective scientific programs, the snapshots are published under a Creative Commons License, available free of charge. The website provides an RSS feed which sends notifications whenever new snapshots appear. The snapshots are also distributed on the mathematics platform www.imaginary.org. Volunteers from the IMAGINARY Community have already begun to translate snapshots into other languages such as German, French, and Spanish. The translated snapshots are again published on the IMAGINARY platform, and announced via social media. 
The snapshots are tagged with one or several Mathematical subjects and possible Connections to other fields are highlighted. This helps visualize the various mathematical areas, their interconnections, and connections to other fields such as biology or physics.

In addition to the snapshot Special values of zeta functions and areas of triangles by Jürg Kramer and AnnaMaria von Pippich appearing in this issue (p. 917), some titles of snapshots so far are: Swarming robots (Magnus Egerstedt), Domino tilings of the Aztec Diamond (Juanjo Rué), and Swallowtail on the shore (Ragnar-Olaf Buchweitz, Eleonore Faber).

\section{Using the Snapshots on Your Campus}

Because snapshots are short, engagingly written, and require very little background, they admit a variety of educational uses. For example, the snapshots provide a way to answer the perennial student questions What is this good for? and Why should I be interested in this? without pointing that student to another textbook. The snapshots give an indication of the variety of mathematical fields and the different flavors they have, so they can be used as an advising tool as well. The snapshots also give a glimpse into what the world of mathematical research is like, so a beginning student who knows she is interested in mathematical research but does not know much about it and is curious can pick up some insights.

A handful of printed snapshots, saddle-stapled, would make a great addition to a recruitment table at a major fair or event targeted at high school students. They can also be shared by your department, college, or university website or social media presence.

\section{Writing a Snapshot}

Snapshots are not quite like the other writing that most mathematicians do on a regular basis. Unlike a journal article, the snapshot is written for readers who may be unfamiliar with not only the mathematical content being described, but also mathematical research in general. Unlike a textbook, the snapshot does not have space for extensive didactics, and the target audience may not have the intrinsic or extrinsic motivation that many textbook users do to keep reading.

One key feature of the snapshots project is an understanding that this style of outreach writing is different from the writing that most mathematicians are very good at doing, so it is reasonable to expect they may need some occasional assistance or advice. That is where the editorial staff comes in: we are available to work with the authors of snapshots on issues of audience, exposition, and appropriateness, in addition to style and formatting.

Some of the particular advice we give to authors applies to mathematical outreach more generally as well:

be modern: Snapshots are about mathematical research, not just exposition about important ideas in mathematics. Most snapshots do contain a historical overview of and background material about the subject, but this should be targeted at bringing the reader up to speed to get an impression of what is going on. keep your audience in mind: Most educated peopleeven those who would go out of their way to read about current mathematical research-have never heard of even such basic notions as a vector space or a number field. These ideas will need to be explained, if not formally defined. As a rule of thumb, it might help to recall what you knew when you were in the earliest stages of your mathematical interest.

trigger prior knowledge to capture the reader: Try to find an intersection of knowledge between you and the target audience and build on something they might know. Is there a good metaphor for an idea you want to explain? Can you think of an everyday analogy or example? You might find it useful to picture someone specific such as your children, your favorite high school teacher, or your first year students, and try to get across to them what your research field is all about. What would fascinate or surprise them? What would you like them to take away?

If the material you present has interesting applications or connections to other areas, mention these in your snapshot-be it in the form of a short paragraph, a reference, a web link, or a picture. If your snapshot focuses on applications of mathematics, please make sure to also present (some aspects of) the mathematics involved, as our readers are very curious to learn about the mathematical methods, as well.

mind the jumps: One of the most common ways the editorial staff helps might be described in terms of continuity: there should not be any large jumps in the level of mathematical sophistication required of the reader. Remember that one small step to you may represent a large jump for nonmathematicians. Try to avoid dramatically changing gears. If you do need to make a leap, tell the reader how they should bridge the gap: Are you generalizing something you explained already? Which aspects remain central, and which are given up? What is the picture the reader should keep in mind?

be comfortable with a bit of informality and imprecision: Remember that the goal is to get the reader to understand the main strokes. For example, formal definitions are not likely to be that helpful to the reader, so you may need to find an informal way to state them. There are some details and hypotheses that may need to be swept under the rug. You can let the reader know you are doing this-it may in fact give you an opportunity to communicate the fact that mathematicians' obsession with detail is one of the forces that drives progress in mathematics. If you would like to label ideas where you are being slightly sloppy, we suggest you use phrases like the following: "The main idea is...," "Neglecting some details,...", "A good picture of the situation is..."

be comfortable with a bit of redundancy and explicitness: Formal mathematical writing is often terse, dense, and minimal, but sometimes in expository writing things need to be spelled out. Saying the same thing in a few different ways may be a waste of space in a journal article, but in a snapshot it may mean 
that the message gets across to and sticks with more readers.

keep it simple, short, vivid, and structured: Your readers are probably not familiar with reading scientific texts. Besides, they might not be native speakers of English. Your readers will find it easier to understand your snapshot if your formulations are

- simple, which means using common terms and sentence structures.

- short, which means using short sentences with few subordinate clauses.

- vivid, which means providing examples and metaphors, using active rather than passive formulations, and expressing your thoughts with verbs rather than with nouns.

- structured, which means providing an overview and a summary, and dividing the text into sections with meaningful subheadings.

be approachable: Approachability is one of the most challenging aspects of writing a snapshot. For example, most journal article abstracts are a simple distillation of the main points of the article. But the abstract of a snapshot is more like an invitation: the abstracts are published as blurbs on the IMAGINARY website. So the abstract is the author's first and best chance to catch a reader's eye. The same reasoning applies to the title of the snapshot. One way to be approachable is to be a bit mysterious.

be respectful: Readers, especially educated readers, can tell when they are being talked down to. A lack of experience with mathematically sophisticated ideas does not entail a lack of experience with sophisticated language, or with other important human endeavors. In fact we expect many of our readers to be interested in and knowledgable about other sciences, philosophy, history, et cetera.

be individual: Many of our snapshots show not only the author's mathematical interests and tastes, but also give a glimpse of their style and personality. Let yourself shine through!

We hope that these suggestions will be useful also for writing about mathematics for a wide audience in general. Maybe you even got interested in writing a snapshot-feel free to contact the organizers of your next meeting when you come to Oberwolfach.

\section{Support and Awards}

The snapshot project was presented at the International Congress of Mathematicians in Seoul on the panel Mathematics communication for the future in 2014 and has been presented at several national and international science communication events, including the International Falling Walls Lab Finals 2015 in Berlin.

The snapshots of modern mathematics from Oberwolfach started as part of the project "Oberwolfach meets IMAGINARY," funded by the Klaus Tschira Foundation. The project is also supported by the Oberwolfach Foundation and the MFO.

\section{Credits}

Photo of Carla Cederbaum courtesy of Friedhelm Albrecht/Universität Tübingen.

Photo of Andrew Cooper courtesy of Andrew Cooper.

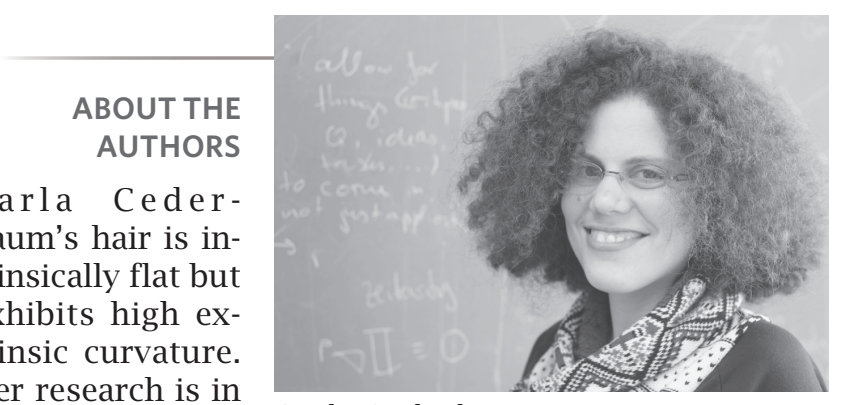

Her research is in

differential geom- Carla Cederbaum

etry, geometric analysis, and mathematical relativity.

Andrew Cooper's primary research is on geometric flows, but his daughter is helping him expand his horizons. 